\title{
Particle observers for state estimation and adaptation in deterministic systems with random piecewise constant inputs
}

\author{
Silvère Bonnabel and Jean-Jacques Slotine
}

\begin{abstract}
In this paper we develop a methodology for state estimation of partially contracting systems inspired by the particle filter (PF). If a system is partially contracting, in the sense that once some state variables are assumed known and viewed as inputs, the remaining reduced system is contracting, then only those variables need to be estimated. Indeed, once those variables are known, the remaining variables are asymptotically recovered owing to the contraction properties. To estimate the state of such a system, we thus suggest to use a PF approach to estimate only the non-contracting part, whereas the other part is naturally recovered. The methodology is applied to a nontrivial tracking problem. The trajectories of objects one seeks to track, such as aircraft and marine vehicles, typically consist of smooth sections with large, but infrequent, unpredictable changes. As a result, their motion is well modeled by nonlinear deterministic ordinary differential equations driven by piecewise constant random control inputs. Following our methodology, we combine a type of PF called the variable rate particle filter (VRPF) with a bank of observers assumed to have convergence properties. Using the notion of virtual system, we devise a hybrid variable rate particle observer, that uses the particles to detect changes, and the observers for state estimation in between changes.
\end{abstract}

\section{INTRODUCTION}

Particle filters have attracted a lot of attention over the past two decades, with the advent of powerful computers, see e.g., [1]. Nowadays, they are able to execute at many hundreds of Hertz for realistic problems, as object tracking (with early implementations dating back to [2]), automotive state estimation, and parameter learning. Virtually all practical implementations of the particle filter resort to the RaoBlackwellized particle filter (RBPF): the state consists of two groups variables $(r, z)$, such that given $r$ the distribution of variable $z$ may be estimated analytically, typically via a Kalman filter. It thus suffices to use particles to estimate $r$, and, using Bayes' theorem the whole distribution of $(r, z)$ is then estimated. This reduces the number of particles and improves estimation accuracy [3].

Building upon this idea, we propose a methodology of particle observers (PO) for partially contracting systems, i.e., systems with state $(r, z)$, and such that if $r(t)$ is known and viewed as an input, the reduced system governing $z(t)$ is deterministic and is contracting, see [4]. Along the lines of the RBPF methodology, the rationale is to use particles to estimate the statistical distribution of variable $r$, whereas $z$ may be asymptotically recovered owing to contraction

S. Bonnabel is with Mines ParisTech, PSL Research University, Centre for Robotics, 60 bd Saint-Michel, 75006 Paris, France silvere.bonnabelemines-paristech.fr

J.J. Slotine is with the Department of Mechanical Engineering and the Department of Brain and Cognitive Sciences, Massachusetts Institute of Technology, Cambridge, MA 02139 USA, jjs@mit. edu properties [5]. This leads to a parsimonious representation of the state, and makes the particle filtering problem more tractable and the filter possibly more accurate.

Intuitively, the main difference is as follows. In the RBPF, $r(\omega, t)$ and $z(\omega, t)$ are stochastic processes, and if $r(\cdot, t)$ is known, then the distribution of $z(\cdot, t)$ is supposed to be analytically computable. In our approach, $z(\cdot, t)$ is deterministically related to $r(\cdot, t)$ owing to the conditionally deterministic assumption. Thus, given $r$ we need to estimate a value for $z(t)$. This is where contraction properties allow us to derive an approximation $\hat{z}(t)$ of this "true" value $z(t)$ instead of an entire distribution, making the use of an observer more suited to estimating the $z$ part of the state.

We then tackle a tracking application. Trajectories of aircraft and marine vehicles are in fact smooth and well described by continuous time ordinary differential equations (ODE) driven by constant inputs, but subject to abrupt changes at each maneuver. Based on this approach to modeling the tracking task, S. Godsill with various co-authors proposed the variable rate particle filter [6], [7], [8], a particle filter well suited to piecewise deterministic models.

On the one hand, it is well known that particle filters have trouble converging in the presence of low process noise, and are subject to divergence when dealing with (even partially) deterministic models, see e.g., [9]. In particular, to the authors best knowledge, the stability of the variable rate particle filter has never been studied. On the other hand, the field of nonlinear observers, and notably the theory of contraction [5], provide powerful tools from dynamical systems theory to achieve stable estimation for noise-free models, but are not suited to tasks such as change detection. This prompts an application of our methodology of particle observers for partially contracting systems. We consider deterministic systems with inputs jumping randomly at unknown times, and run many observers in parallel (the particles), each assumed to be contracting in the absence of jumps. This way, we obtain a hybrid filter which is able to detect the jumps, and to reconverge to the true state between jumps. The theory is made rigorous using the notion of virtual systems introduced in [4], see also [10]. Our approach may be related to the flow particle filter [11], and the feedback particle filter [12]. Some preliminary ideas can be found in our recent paper [13].

Section II is devoted to the particle observer methodology. Section III considers the piecewise deterministic estimation problem, and the variable rate particle filter is introduced. In Section IV, assuming we have at our disposal an observer with some convergence properties, a hybrid strategy that 
combines it with a particle filter is proposed.

\section{Particle filters And Particle observers}

\section{A. The Rao-Blackwellized particle filter (RBPF)}

Consider a discrete Markov process $r_{n}$ having initial distribution $p\left(r_{0}\right)$ and transition kernel $p\left(r_{n} \mid r_{n-1}\right)$. The variable $r_{n}$ is supposed hidden, and assume we have observations in the form of a random variable $y_{n}$ at time $n$ correlated with $r_{n}$. Moreover, assume the observations to be conditionally independent given the hidden variables. The goal of discrete time filtering is to estimate the hidden variables at best from the observations, i.e., to ideally compute

$$
p\left(r_{n} \mid y_{1: n}\right), \quad \text { where } y_{1: n}=\left\{y_{1}, \cdots, y_{n}\right\},
$$

or more generally $p\left(r_{1: n} \mid y_{1: n}\right)$, a task known as smoothing. Assume now, that observations involve another process $z_{n}$, such that $p\left(z_{n} \mid y_{1: n}, r_{1: n}\right)$ may be analytically evaluated, typically using a Kalman filter.

If we may approximate the desired distribution using a set of weighted particles, i.e., $p\left(r_{1: n} \mid y_{1: n}\right) \approx \sum_{1}^{N} w_{n}^{(i)} \delta_{r_{1: n}^{(i)}}\left(r_{1: n}\right)$, then the best estimate based on past observations $y_{1: n}$ of any quantity $F\left(z_{n}, r_{n}\right)$ in the sense of minimal mean squared error may be approximated as:

$$
\mathbb{E}_{p\left(z_{n}, r_{n} \mid y_{1: n}\right)}\left(F\left(z_{n}, r_{n}\right)\right) \approx \sum_{i=1}^{N} w_{n}^{(i)} \mathbb{E}_{p\left(z_{n} \mid y_{1: n}, r_{1: n}^{(i)}\right)}\left(F\left(z_{n}, r_{n}^{(i)}\right)\right)
$$

where we used $p\left(z_{n}, r_{1: n} \mid y_{1: n}\right)=p\left(z_{n} \mid r_{1: n}, y_{1: n}\right) p\left(r_{1: n} \mid y_{1: n}\right)$.

This gives rise to the celebrated Rao-Blackwellized Particle Filter (RBPF). Algorithm 1 provides its basic version.

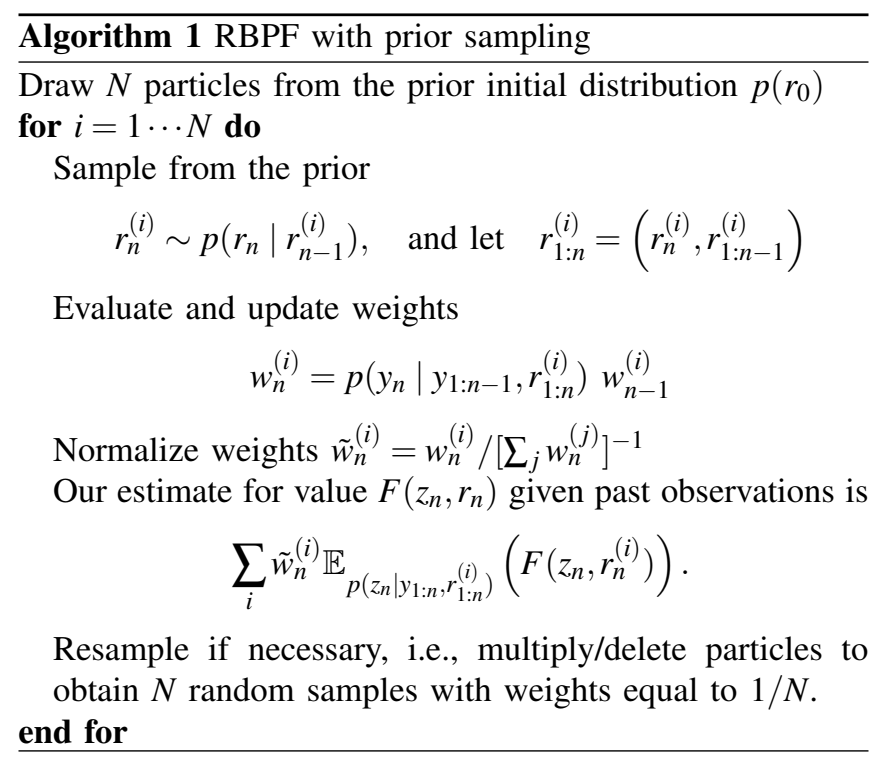

\section{B. Particle observers for partially contracting systems}

In this paragraph, we summarize the results of [13]. Consider a noisy dynamical system of the form

$$
\begin{aligned}
& \frac{d}{d t} z=f(z, r) \\
& \frac{d}{d t} r=g(r)+w(t)
\end{aligned}
$$

where $f, g$ are smooth maps, and $w(t)$ process noise.

Definition 1: If equation (2) is a contraction when $r(t)$ is considered as a known input, system (2)-(3) is said to be a partial contraction [4].

This implies, see [5], that if $r(t)$ is known and viewed as an input of (2), then (2) has the property that for any two trajectories $z_{1}(t), z_{2}(t)$ we have:

$$
d\left(z_{1}(t), z_{2}(t)\right) \leq d\left(z_{1}(0), z_{2}(0)\right) e^{-2 \lambda t},
$$

with $\lambda$ the contraction rate, and $d$ a well-chosen distance.

Assume discrete time noisy measurements are available:

$$
y_{n}=h\left(z\left(t_{n}\right), r\left(t_{n}\right)\right)+V_{n} \quad \text { at times } t_{0}<t_{1}<t_{2}<\cdots,
$$

with $V_{n}$ unknown independent identically distributed random variables with density $l$, i.e., $p(y \mid z, r)=l(y-h(z, r))$.

Note that, by denoting $\left(r_{n}, z_{n}\right):=\left(r\left(t_{n}\right), z\left(t_{n}\right)\right)$ one may want to apply the RBPF methodology to dynamics (2)-(3) with observations (5). However, the usual assumption underlying the RBPF that $p\left(z_{n} \mid y_{1: n}, r_{1: n}\right)$ may be analytically evaluated does not hold. It may yet be approximated under the partial contraction assumption introduced in Definition 1. Indeed, if we call $\hat{z}(t)$ a solution of (2) associated to some given trajectory $\{r(t)\}_{t \geq 0}$, then asymptotically we have $p(z(t) \mid$ $\left.\{r(s)\}_{s \leq t}\right) \approx \delta(z(t)-\hat{z}(t))$, since (4) implies exponential convergence of $\hat{z}(t)$ to $z(t)$. Thus, letting $\left(\hat{z}(t)^{(i)}, r(t)^{(i)}\right)$ be a solution to the stochastic differential equations (SDE) (2)-(3), we have the approximations:

$$
\begin{aligned}
& p\left(z(t) \mid y_{1: n},\left\{r(s)^{(i)}\right\}_{s \leq t}\right) \approx \delta\left(z_{t}-\hat{z}(t)^{(i)}\right), \quad t \geq t_{n} \\
& \mathbb{E}_{p\left(z(t) \mid y_{1: n},\left\{r(s)^{(i)}\right\}_{s \leq t}\right)}\left(F\left(z(t), r(t)^{(i)}\right)\right) \approx F\left(z(t)^{(i)}, r(t)^{(i)}\right), \\
& p\left(y_{n} \mid y_{1: n-1},\left\{r(s)^{(i)}\right\}_{s \leq t_{n}}\right) \approx l\left(y_{n}-h\left(\hat{z}\left(t_{n}\right)^{(i)}, r\left(t_{n}\right)^{(i)}\right)\right) .
\end{aligned}
$$

An application of the RBPF methodology to (2)-(3) with observations (5) under the partial contraction assumption and the subsequent approximations (6)-(7) thus yields the particle observer (PO) Algorithm 2.

\footnotetext{
Algorithm 2 The $\mathrm{PO}$ with prior sampling

Draw $N$ particles from the prior initial distribution $p\left(z_{0}, r_{0}\right)$ for $i=1 \cdots N$ do

Sample $\left(z\left(t_{n}\right)^{(i)}, r\left(t_{n}\right)^{(i)}\right)$ by numerically integrating the stochastic differential equations (2)-(3) from $t_{n-1}$ to $t_{n}$. Evaluate and update weights

$$
w_{n}^{(i)}=l\left(y_{n}-h\left(z\left(t_{n}\right)^{(i)}, r\left(t_{n}\right)^{(i)}\right)\right) w_{n-1}^{(i)} .
$$

Normalize weights $\tilde{w}_{n}^{(i)}=w_{n}^{(i)} /\left[\sum_{j} w_{n}^{(j)}\right]^{-1}$.

Our estimate for value $F(z, r)$ given past observations is

$$
\sum_{i} \tilde{w}_{n}^{(i)} F\left(r(t)^{(i)}, z(t)^{(i)}\right) .
$$

In particular the state estimate is $\sum_{i} \tilde{w}_{n}^{(i)}\left(r(t)^{(i)}, z(t)^{(i)}\right)$.

Resample if necessary.

\section{end for}




\section{STATE ESTIMATION FOR DETERMINISTIC SYSTEMS WITH RANDOM JUMPS}

The reader might wonder to what extent it is realistic to consider systems of the form (2)-(3) where noise affects only (3). First, it may serve as a meaningful approximation, if for instance (2) is subject to small noise. Then, and above all, it may readily be applied to various realistic systems. One example consists of nonlinear piecewise deterministic systems subject to abrupt unpredictable jumps. These are relevant to tracking applications, and in the remainder of this paper we apply the ideas of Section II to such systems. Various other perspectives are offered in Section V.

\section{A. Original considered model}

In this section we review the conditionally deterministic inference problem addressed by the variable rate particle filter [6], see also [8] for a somehow more concise presentation. We consider a deterministic dynamical model with continuous-time state $x(t) \in \mathbb{R}^{n}$, and parameter (or control input) $u \in \mathbb{R}^{m}$. We assume that changepoints occur at random times $\left\{\tau_{0}=0, \tau_{1}, \cdots, \tau_{k}, \cdots\right\}$, each with associated parameters $\left\{u_{0}, u_{1}, \cdots, u_{k}, \cdots\right\}$. The pairs $\left(\tau_{k}, u_{k}\right)$ form elements of a marked point process. Consider some discrete measurement times $t_{0}, t_{1}, \cdots, t_{n}, \cdots$, along with associated measurements $y_{0}, y_{1}, \cdots, y_{n}, \cdots$ of the form

$$
y_{n}=h\left(x\left(t_{n}\right)\right)+V_{n}
$$

with $V_{n}$ a random variable with known distribution. By letting $l$ denote the probability density function of $V_{n}$ we have

$$
p\left(y_{n} \mid x\left(t_{n}\right)\right)=l\left(y_{n}-h\left(x\left(t_{n}\right)\right)\right) .
$$

The objective is to estimate from partial and noisy measurements of $x(t)$, the changepoints, the associated parameters, and the state $x(t)$ in real time.

Let $\theta_{n}$ represent the past and present variables

$$
\theta_{n}=\left\{\tau_{1}, \cdots, \tau_{j}, u_{1}, \cdots, u_{j}: 0 \leq \tau_{j}<t_{n} \leq \tau_{j+1}\right\} .
$$

Let us define the following quantities

$$
K(t)=\max \left\{k: \tau_{k}<t\right\}, \quad K_{n}=K\left(t_{n}\right)
$$

Note that, we could alternatively write $K_{n}=\tilde{K}\left(\theta_{n}\right)$ as

$$
K_{n}=\max \left\{i: \tau_{i} \in \theta_{n}\right\}
$$

Processes $\tau_{k}$ and $u_{k}$ are assumed to be Markov, and the transitions to be given by

$$
p\left(\tau_{k} \mid \tau_{k-1}\right)=p_{\tau}\left(\tau_{k}-\tau_{k-1}\right), \quad p_{u}\left(u_{k} \mid u_{k-1}\right)
$$

with $p_{\tau}$ a probability density such that $p_{\tau}(\tau)=0$ for $\tau<0$.

The state evolution is assumed to be governed by an ODE which depends on the changepoints and the corresponding parameters as follows:

$$
\frac{d}{d t} x(t)=f\left(x(t), t, u_{K(t)}\right) .
$$

This means that the state is governed by an ODE driven by parameters that remain constant over random time intervals. Let us denote $\phi$ the flow of the ODE $\dot{x}=f(x, t, u)$, that is, $\phi\left(x_{1}, u, t_{1}, t_{2}\right)$ is the solution at $t_{2}$ to the latter ODE with fixed parameter $u$ and initial condition $x_{1}$ at $t_{1}$. We have:

$$
x(t)=\phi\left(x\left(\tau_{K_{n}}\right), u_{K_{n}}, \tau_{K_{n}}, t\right), \quad \tau_{K_{n}}<t \leq \tau_{K_{n}+1} .
$$

By choosing $t=\tau_{K_{n}+1}$, this equation specifies the state at the next changepoint, and by reiterating to the following changepoints, we get the whole future trajectory. This proves that the knowledge of the process $\theta_{n}$ and the initial state $x(0)$ uniquely specifies the state $x(t)$. For simplicity, we will systematically assume $x(0)$ to be known. This is not a very restrictive assumption, as the aim of tracking is to keep track of $x(t)$ despite the random changepoints, and after a few jumps this knowledge about the initial state is forgotten.

Recalling (1), the goal of the variable rate particle filter is to compute an approximation to the posterior

$$
p\left(\theta_{n} \mid y_{1: n}\right)
$$

and $x(t)$ as a byproduct, through (12), i.e. by integrating (11).

\section{B. The variable rate particle filter}

The bootstrap variable rate particle filter (VRPF) is a particle filter that computes an approximation

$$
\hat{p}\left(\theta_{n} \mid y_{1: n}\right)=\sum_{j=1}^{N} w_{n}^{(j)} \delta_{\theta_{n}^{(j)}}\left(\theta_{n}\right)
$$

to the posterior (13). In its simplest bootstrap form, the piecewise deterministic VRPF is implemented along the lines of Algorithm 1, see [8], yielding Algorithm 3.

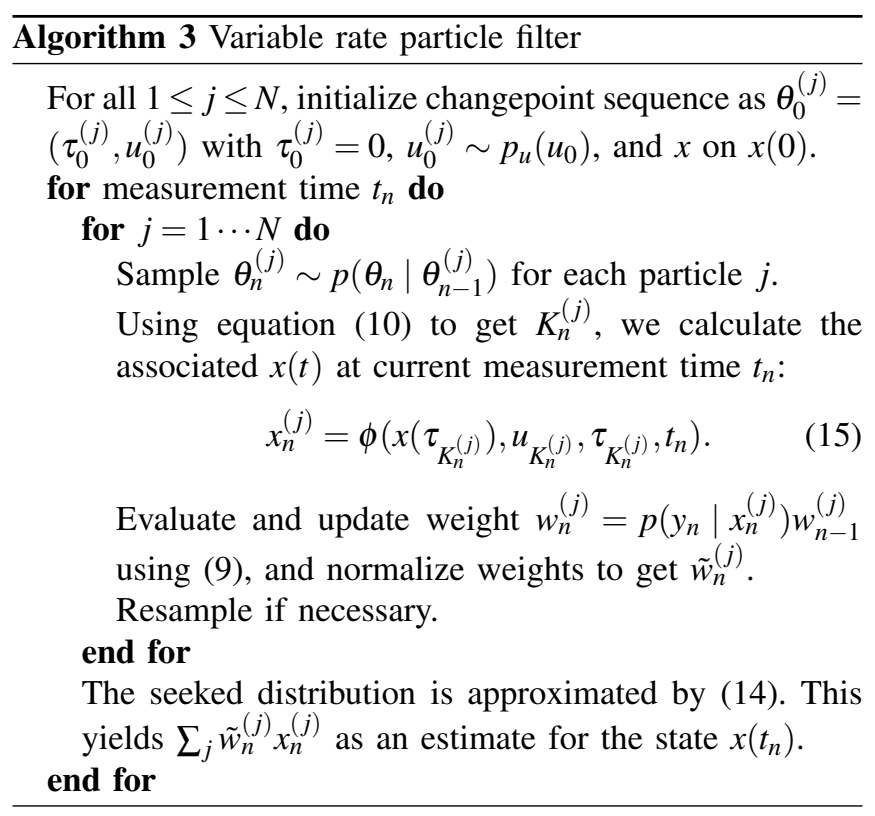

Let us now comment on some of the steps. Regarding the sampling step, assume that we want to sample $\tau_{K_{n}}$ given $\tau_{K_{n-1}^{(j)}}$. This means there has been exactly $K_{n-1}^{(j)}$ changepoints before $t_{n-1}$. We can sample then the next changepoint time as $\tau_{K_{n-1}^{(j)}+1} \sim p_{\tau}\left(\tau-\tau_{K_{n-1}^{(j)}}\right)$. Either $t_{n} \leq \tau_{K_{n-1}^{(j)}+1}$, then we let $\bar{\tau}$ denote the latter time and we let $K_{n}^{(j)}=K_{n-1}^{(j)}$. $\theta$ does not 
need to be sampled anymore until the first measurement time $t_{k}, k>n$ to become larger than $\bar{\tau}$. Or $\tau_{K_{n-1}^{(j)}+1}<t_{n}$, then we integrate the flow (12) to obtain $x^{(j)}\left(\tau_{K_{n-1}^{(j)}+1}\right)$, then sample $u_{K_{n-1}^{(j)}+1} \sim p_{u}\left(u \mid u_{K_{n-1}^{(j)}}\right)$, and reiterate until the changepoint time becomes larger than $t_{n}$.

Regarding the weight update, particles are sampled using the prior and the weights are classically defined as $w_{n}^{(j)}=p\left(y_{n} \mid \theta_{n}^{(j)}, y_{1: n-1}\right) w_{n-1}^{(j)}$, see Algorithm 1. As $x(0)$ is known (and thus considered as a fixed parameter), given the sequence $\theta_{n}^{(j)}$ of changepoint times and parameters, the state corresponds to the integration of system (11), that is $x_{n}^{(j)}$ as defined by (15). Using that $x_{n}^{(j)}$ is a function $\mathscr{F}\left(\theta_{n}^{(j)}\right)$ of the past changepoints we have $p\left(y_{n} \mid \theta_{n}^{(j)}, y_{1: n-1}\right)=$ $p\left(y_{n} \mid \theta_{n}^{(j)}, \mathscr{F}\left(\theta_{n}^{(j)}\right), y_{1: n-1}\right)=p\left(y_{n} \mid x_{n}^{(j)}\right)$ indeed, as $y_{n}$ is independent of all the other variables conditionally on $x_{n}$.

\section{Possible caveats in a partially deterministic context}

It is well known that particle filters are not efficient for systems governed by deterministic dynamics, typically for fixed parameter inference. Actually, they are prone to divergence, see e.g., [9]. In the context of the VRPF, though, the state space is continually explored thanks to the jumps. But asymptotic convergence may necessitate a large number of particles to get full coverage. Another possible limitation of $\mathrm{PF}$ is in the presence of low measurement noise. Since $p\left(y_{n} \mid x_{n}\right)$ is then peaked, virtually all weights drop to zero after one measurement, leading in turn to estimation failure.

\section{A PARTICLE OBSERVER BASED APPROACH}

In the present section, we consider the model of Section III-A, and propose a particle observer, that addresses some of the issues raised in Section III-C.

Up to a few subtleties related to marked point processes in place of discrete-time Markov processes, Algorithm 3 is close in essence to the standard Algorithm 1, since $\theta=(\tau, u)$ plays the role of hidden variable $r$, and $x$ plays the role of auxiliary variable $z$, see notably the discussion after Algorithm 3. As a result, one may want to pursue the methodology of particle observers described at Section II-B for this application. Our idea is that, indeed, if the sequence of jump times $\tau_{1}, \tau_{2}, \cdots$ were known, then an asymptotic observer might be devised to estimate the remaining variables, that is, $x(t)$ and input $u(t)$. And under contraction properties of the observer's dynamics, approximations akin to (6)-(7) may then be used. To formalize this approach, we will need to split the observations in two. One type of observation will be used by the observer, and the other type by the particle filter to assess the weights. We will assume to have discrete the noisy measurements (8), but also some auxiliary continuous-time noise-free measurements of the form:

$$
z(t)=\tilde{h}(x(t)) \text {. }
$$

Note that the two sources of information are assumed independent, and $h$ and $\tilde{h}$ may bear a quite different structure. For instance, $z(t)$, albeit noise free, may be of very small dimension compared to $x(t)$.

\section{A. Considered problem and further assumptions}

To recap, the considered system is

$$
\begin{aligned}
\frac{d}{d t} x(t) & =f\left(x(t), t, u_{K(t)}\right) \\
y_{n} & =h\left(x\left(t_{n}\right)\right)+V_{n}, \quad t_{1}<t_{2}<\cdots \\
z(t) & =\tilde{h}(x(t))
\end{aligned}
$$

where $u_{K}(t)$ are parameters jumping at random times. Besides, let us consider the following alternative system

$$
\begin{aligned}
\frac{d}{d t} x(t) & =f(x(t), t, u(t)) \\
\frac{d}{d t} u(t) & =0 \\
z(t) & =\tilde{h}(x(t))
\end{aligned}
$$

which corresponds to the noise free continuous-time system (17)-(19) between two jumps (as the parameters are fixed between jumps). Furthermore, assume we have at our disposal an asymptotic observer for the latter system of the form:

$$
\begin{aligned}
& \frac{d}{d t} \hat{x}=f(\hat{x}, t, \hat{u})+L_{1}(t, \hat{x}, \hat{u})(z-\tilde{h}(\hat{x})) \\
& \frac{d}{d t} \hat{u}=L_{2}(t, \hat{x}, \hat{u})(z-\tilde{h}(\hat{x}))
\end{aligned}
$$

having asymptotic convergence properties for arbitrary initial condition $(\hat{x}(0), \hat{u}(0))$. Typically, a desirable property would be exponential contraction [5], leading to

$$
d(\hat{x}(t), x(t)) \leq d(\hat{x}(0), x(0)) e^{-2 \lambda t} .
$$

On the one hand, this observer can readily be used on the original problem (17)-(19). This will lead to a solution that is unlikely to diverge (provided average time between consecutive jumps is larger than the convergence time of the observer), but in the transitory phases that follow changepoints, it will yield inaccurate estimates until $(\hat{x}(t), \hat{u}(t))$ has re-converged to $\left(x(t), u_{K}(t)\right)$.

On the other hand, the variable rate particle filter cannot readily account for the information provided by $z(t)$ of eq. (19), since noise free measurements (as they appear in the theory of observers), or very small measurement noise, lead to failure of particle filters as explained in Section IIIC. Besides, owing to the fact the dynamics are piecewise deterministic, the variable rate particle filter based solely on the information brought by $y_{n}$ of eq. (18) will never be able to guarantee asymptotic convergence to the true parameters, even if the time between jumps is large (as the particles $u^{(j)}$ remain fixed between two jumps). For those reasons, we propose a hybrid strategy, combining both approaches.

\section{B. Proposed hybrid strategy}

Our idea is to devise a variable rate particle filter for system (17)-(18), and to use the side partial information (19) to help the sampled parameters $u^{(j)}$ (and thus the state) move towards the "true" parameters and state between consecutive changepoints. Crucially, this can be done by resorting to the notion of virtual systems [4], [10], i.e., by applying the variable rate methodology to an auxiliary virtual system. 
Consider indeed the following virtual system associated to the observer

$$
\begin{aligned}
& \frac{d}{d t} \chi=f(\chi, t, v)+L_{1}(t, \chi, v)(z-\tilde{h}(\chi)) \\
& \frac{d}{d t} v=L_{2}(t, \chi, v)(z-\tilde{h}(\chi))
\end{aligned}
$$

and rewrite those dynamics as

$$
\frac{d}{d t}(\chi, v)=\hat{f}(\chi, v, t, z)
$$

Building upon the latter systems, we let $z(t)=\tilde{h}(x(t))$ as before, and we define auxiliary dynamics (recall that $\tau_{1}, \tau_{2}, \cdots, u_{1}, u_{2} \cdots$ are in fact unknown) as

$$
\begin{aligned}
\tau_{k-1}<t<\tau_{k} & \Rightarrow \frac{d}{d t}(\chi, v)=\hat{f}(\chi, v, t, z), \\
t=\tau_{k} & \Rightarrow v(t)=u_{k},
\end{aligned}
$$

that is, we integrate (28) between two consecutive changepoints, and, at each changepoint at time $\tau_{k}$, the variable $v$ jumps to the corresponding parameter value $u_{k}$.

Letting $\tilde{\phi}$ denote the flow of (28), i.e., $\tilde{\phi}\left(\chi_{1}, v_{1}, t_{1}, z^{\left(t_{1}, t_{2}\right)}, t_{2}\right)$ is the solution at $t_{2}$ to (28) being initialized on $\left(\chi_{1}, v_{1}\right)$ at $t_{1}$, and where we let $z^{\left(t_{1}, t_{2}\right)}=\left(\tilde{h}(x(s))_{t_{1} \leq s \leq t_{2}}\right.$. Our auxiliary virtual system can be alternatively defined by

$$
\begin{aligned}
(\chi(t), v(t)) & =\tilde{\phi}\left(\chi\left(\tau_{K_{n}}\right), v\left(\tau_{K_{n}}\right), \tau_{K_{n}}, z^{\left(\tau_{K_{n}}, t\right)}, t\right), \tau_{K_{n}}<t \leq \tau_{K_{n}+1} \\
y_{n} & =h\left(\chi\left(t_{n}\right)\right)+V_{n}, \quad t_{1}<t_{2}<\cdots
\end{aligned}
$$

that is, we recover an auxiliary system to which the variable rate methodology of Section III-B can be applied. This yields the proposed Algorithm 4.

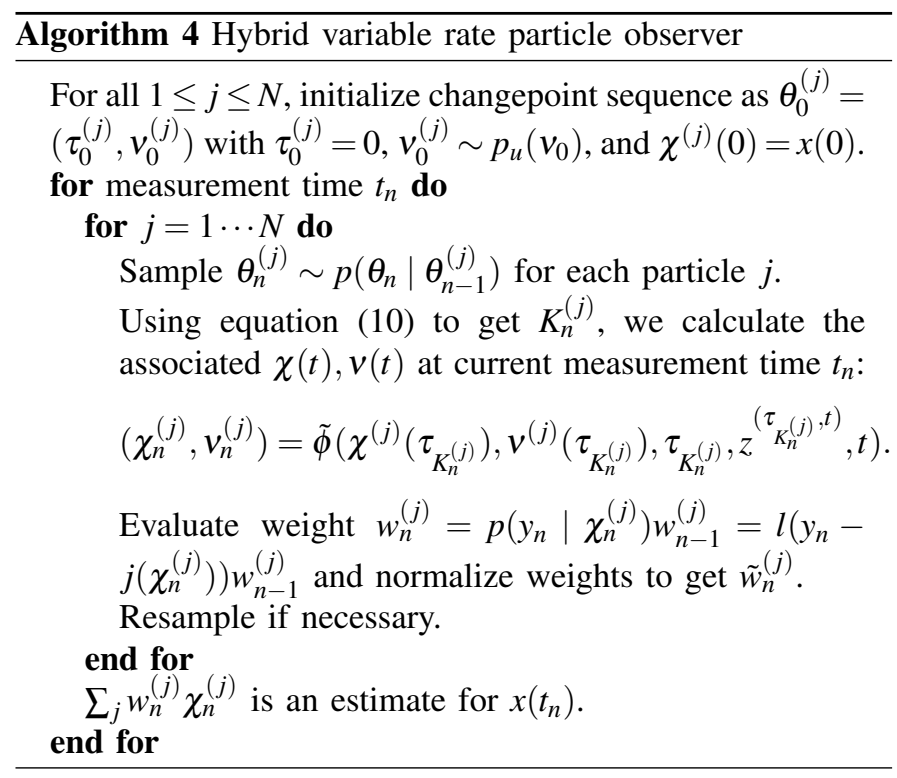

Using the flow of auxiliary system (26)-(27) in place of the flow of original system (17) to estimate the state of the original system through a VRPF, may be justified through the notion of virtual system, see Section IV-C below.
Remark 1: Particle filters have drawn criticism regarding their execution time. In this respect, our method is particularly efficient, owing to the use of observers as building blocks, each being very cheap to implement, as for each particle we need to integrate an ODE and weight computation is also cheap since $p(y \mid \chi)=l(y-h(\chi))$, see (9). This is in contrast to the standard RBPF where Kalman filters are commonly used to estimate the $z$ variable, see Section II.

\section{Discussion and formal results}

System (17)-(18)-(19) is stochastic, since it is driven by the process $\omega \mapsto\left(\tau_{k}(\omega), u_{k}(\omega)\right)$, and $\omega \mapsto V_{n}(\omega)$ is a random variable. Notably, $z(t)$ is a random process $\omega \mapsto z(t, \omega)$. Based on the fact that in the VRPF methodology the initial condition $x(0)$ is assumed to be known, the following result is noteworthy:

Lemma 1: Consider system (17)-(19) on the one hand, and system (29) on the other. Then, if $\chi(0)=x(0)$, we have $\chi(t)=x(t)$ at all times $t \geq 0$.

Proof: It suffices to note that, since $z(t)=\tilde{h}(x(t))$, we have $z(t)-\tilde{h}(x(t))=0$, and the pair $\left(x(t), u_{K(t)}\right)$ is thus a solution to the stochastic differential equation (29).

This property lies at the heart of the use of virtual systems for observer design [4], [10]. In terms of our particle filter approach, this means Algorithm 4 may be viewed as a (bootstrap variable rate) particle filter for the original problem, given that initial conditions coincide $\chi(0)=x(0)$. Indeed, in this case both dynamical systems coincide as explained in the proof of Lemma 1. Normally, Algorithm 4 should use observations related to its own latent variables, i.e., of the form $\tilde{y}_{n}=h\left(\chi\left(t_{n}\right)\right)+V_{n}$, whereas it evaluates the weights based on the measurements $y_{n}=h\left(x\left(t_{n}\right)\right)+V_{n}$ instead. However, it is legitimate to use $y_{n}$ as measurements for the auxiliary system since we know from Lemma 1 that at all times $\chi(t)=x(t)$ and thus $\tilde{y}_{n}=y_{n}$ indeed.

However, Lemma 1 does not hold under an arbitrary small error in measurements described by (19), or for $\chi(0) \neq$ $x(0)$. But those are precisely the cases where our observerbased approach applies. Indeed, by assuming observer (23)(24) to have convergence properties, each simulated particle $\left(\chi^{(j)}(t), v^{(j)}(t)\right)$ obtained through $\tilde{\phi}$ in Algorithm 4 tends to move closer to $\left(x(t), u_{K(t)}\right)$ between jumps. As a result, particles tend to be less dispersed around $x(t)$ and to yield more precise and robust estimates, see Fig. 1 for an illustration.

\section{Generalizations}

Note that Eq. (17) being quite general, our approach can be used to enhance existing adaptive observers to track more rapidly variations in some unknown parameters $u$ subject to abrupt changes.

Another possibility is to let $u$ denote a model index, and then $\tau$ denotes the unknown times of switching.

Besides, the assumption (21) of constant $u(t)$ between jumps is not necessary. Between jumps, generic dynamical behavior for $u(t)$ may in fact be assumed as long as it is contracting or may be tracked by an observer. In this case $u_{K(t)}$ in (17) shall be replaced with $u_{K(t)}(t)$ and (21) by the ad hoc differential equation $\frac{d}{d t} u(t)=g(u(t), t)$. 


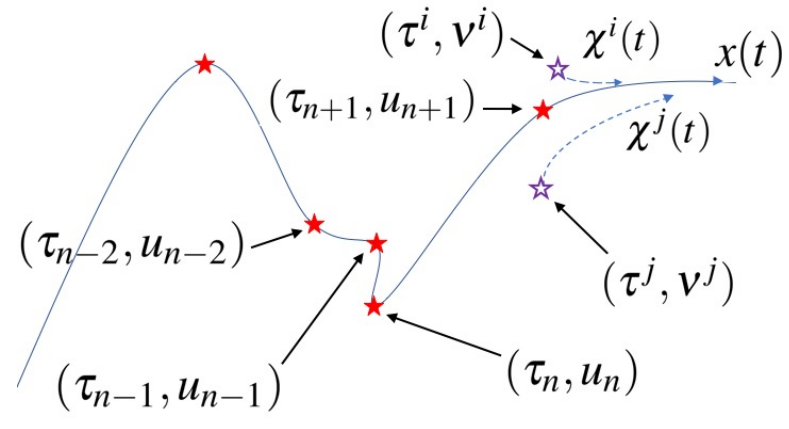

Fig. 1. Graphical illustration of Algorithm 4 with $x(t) \in \mathbb{R}^{2}$. Plain stars indicate changing points at unknown times $\tau_{1}, \tau_{2}, \cdots$, each being associated with unknown parameter $u_{i}$. The particle observer of Algorithm 4 keeps exploring the space of jump times $\tau$ and parameter $u$ by constantly creating tentative couples $\left(\tau^{j}, v^{j}\right)$ with associated variable $\chi^{j}(t)$ referred to as particles, and makes use of a great number $N$ of such particles. Owing to the assumed convergence properties of observer (23)-(24) for each particle $1 \leq j \leq N$, variable $\chi^{j}(t)$ tends to move towards true $x(t)$ even if the corresponding tentative couple $\left(\tau^{j}, v^{j}\right)$ is misidentified. The philosophy of particle filtering, that has been compared to Darwin's "survival of the fittest", consists in evaluating weights $w^{j}$ for each particle indicating its fit to measured data. Particles with low weights are discarded, and particles with high weight are multiplied to populate the likely regions of the space, yielding quicker convergence. Here particle $i$ corresponds to a tentative $\left(\tau^{i}, v^{i}\right)$ being close to actual $\left(\tau_{n+1}, u_{n+1}\right)$ and thus $\chi^{i}(t)$ rapidly converges to the true $x(t)$. Particle $i$ is thus likely to be selected and multiplied, whereas particle $j$ having less adequate $\left(\tau^{j}, v^{j}\right)$ might be suppressed.

\section{PERSPECTIVES}

Building upon the notion of virtual systems [4], we proposed a novel method which bridges the gap between nonlinear observer theory and particle filters for a broad class of practical applications.

We anticipate the method might prove useful in various contexts. One such instance is target tracking using one particular model of the nonlinear deterministic models with random piecewise constant inputs proposed in [7]. For a 3D target model inspired by [7], an invariant extended Kalman filter based on the work [14] was shown in [15] to yield good tracking performance, and might be used as a building block in a particle observer approach.

The method can also be applied to adaptive estimation of deterministic systems parameterized by an unknown vector selected from a finite set of known vectors. The switch is randomly connected to one of the vectors and may either remain there throughout the duration of the process, or change at random times associated to a prior temporal distribution. A typical example application would be Simultaneous Localization and Mapping (SLAM) with a vector accounting for the multi-hypotheses that stem from perceptual ambiguity, as e.g. in [16]. To address such questions one could combine the contracting observer of [17], based on the construction of simple synthetic measurements for SLAM, with the particle approach developed in this article. Another possible application is contact detection in robotics, see also Section IV-D. In the recent paper [18] for instance, leg state switching is based on a periodic phase-based state schedule, which provides a prior on times of change of a contact parameter $s$ between stance $(s=0)$ and swing $(s=1)$.
Disturbance observers [18] with convergence properties for known $s$ might be run in parallel using our approach.

The methodology of particle observers might also prove useful in the context of differentially positive systems [19], where the state is known to converge to a Perron-Frobenius curve: the use of particles might allow us to identify accurately where the state is located on the curve.

\section{ACKNOWLEDGEMENTS}

We thank Prof. Godsill for useful discussions, while the first author was visiting the University of Cambridge as a fellow of Sidney Sussex College. The authors would also like to thank Marion Pilté and Frédéric Barbaresco from the company Thales Air Systems for fruitful discussions.

\section{REFERENCES}

[1] A. Doucet, S. Godsill, and C. Andrieu, "On sequential Monte Carlo sampling methods for bayesian filtering," Statistics and Computing, vol. 10, no. 3, pp. 197-208, 2000.

[2] P. D. Moral, J. Noyer, G. Rigal, and G. Salut, "Particle resolution and non-linear signal processing with radar/sonar application," Traitement du Signal, vol. 12, no. 4, pp. 287-302, 1995.

[3] A. Doucet, N. De Freitas, K. Murphy, and S. Russell, "RaoBlackwellised particle filtering for dynamic bayesian networks," in Proceedings of the Sixteenth conference on Uncertainty in artificial intelligence. Morgan Kaufmann Publishers Inc., 2000, pp. 176-183.

[4] W. Wang and J.-J. E. Slotine, "On partial contraction analysis for coupled nonlinear oscillators," Biological cybernetics, vol. 92, no. 1, pp. 38-53, 2005.

[5] W. Lohmiller and J.-J. E. Slotine, "On contraction analysis for nonlinear systems," Automatica, vol. 34, no. 6, pp. 683-696, 1998.

[6] S. Godsill and J. Vermaak, "Variable rate particle filters for tracking applications," in IEEE/SP 13th Workshop on Statistical Signal Processing, 2005. IEEE, 2005, pp. 1280-1285.

[7] P. Bunch and S. Godsill, "Dynamical models for tracking with the variable rate particle filter," in Information Fusion (FUSION), 2012 15th International Conference on. IEEE, 2012, pp. 1769-1775.

[8] — , "Particle smoothing algorithms for variable rate models," IEEE Trans. on Signal Processing, vol. 61, no. 7, pp. 1663-1675, 2013.

[9] N. Kantas, A. Doucet, S. S. Singh, J. Maciejowski, N. Chopin et al., "On particle methods for parameter estimation in state-space models," Statistical science, vol. 30, no. 3, pp. 328-351, 2015.

[10] J. Jouffroy and J.-J. Slotine, "Methodological remarks on contraction theory," in Decision and Control, 2004. CDC. 43rd IEEE Conference on, vol. 3. IEEE, 2004, pp. 2537-2543.

[11] F. Daum, "Nonlinear filters: beyond the Kalman filter," IEEE Aerospace and Electronic Systems Magazine, vol. 20, no. 8, pp. 57-69, 2005

[12] T. Yang, P. G. Mehta, and S. P. Meyn, "Feedback particle filter," IEEE Trans. on Automatic Control, vol. 58, no. 10, pp. 2465-2480, 2013.

[13] S. Bonnabel and J.-J. Slotine, "Particle observers for contracting dynamical systems," in International Conference on Geometric Science of Information. Springer, 2017, pp. 310-317.

[14] A. Barrau and S. Bonnabel, "The invariant extended Kalman filter as a stable observer,' IEEE Transactions on Automatic Control, vol. 62, no. 4, pp. 1797-1812, 2017.

[15] M. Pilté, S. Bonnabel, and F. Barbaresco, "Tracking the Frenet-Serret frame associated to a highly maneuvering target in 3D," in Decision and Control (CDC), IEEE 56th Conference on, 2017, pp. 1969-1974.

[16] I. Kanitscheider and I. Fiete, "Training recurrent networks to generate hypotheses about how the brain solves hard navigation problems," in NIPS, 2017, pp. 4529-4538.

[17] F. Tan, W. Lohmiller, and J.-J. Slotine, "Analytical SLAM without linearization," The International Journal of Robotics Research, vol. 36, no. 13-14, pp. 1554-1578, 2017.

[18] G. Bledt, P. M. Wensing, S. Ingersoll, and S. Kim, "Contact model fusion for event-based locomotion in unstructured terrains," in International Conf. on Robotics and Automation (ICRA), 2018, pp. 1-8.

[19] F. Forni and R. Sepulchre, "Differentially positive systems," IEEE Trans. on Automatic Control, vol. 61, no. 2, pp. 346-359, 2016. 